\title{
A condition-based maintenance policy for a two-unit system subject to dependent soft and hard failures: A reinforcement learning approach
}

\author{
Seyedvahid Najafi*, Ji Ye Janet Lam**, Chi-Guhn Lee*** \\ Department of Mechanical and Industrial Engineering, University of Toronto, ON M5S3G8, Canada \\ *vahid.najafi@mail.utoronto.ca \\ **janet@mie.utoronto.ca \\ ***cglee@mie.utoronto.ca
}

\begin{abstract}
Maintenance planning plays an essential role in improving the reliability of a system and its effectiveness. Condition-based maintenance (CBM) suggests preventive actions to avoid failures, considering the state of a system. In this paper, an opportunistic CBM policy is developed for a series system composed of two units whose conditions are monitored regularly. The reliability functions are obtained using the proportional hazards model (PHM) to estimate the system's remaining useful life. When the deterioration of a unit exceeds a predefined threshold, a soft failure occurred, which can be detected through inspection. Hard failures are rectified by a corrective action immediately, including minimal repair, general repair, and replacement. The objective is to find an optimal policy that minimizes the total expected cost of the system. The problem is formulated in the semi-Markov decision process (SMDP) framework, and a reinforcement learning algorithm is proposed to find the optimal control policy and the long-run expected average cost per unit time. This study extends previous models, suggesting a new opportunistic CBM policy using the PHM in which actions with different levels can be performed on a two-unit series system.
\end{abstract}

\section{Introduction}

Manufacturing systems are composed of multiple components that interact with each other in different ways. The components wear out with age and usage, and lack of an appropriate asset management plan can incur considerable operation and maintenance (O\&M) costs and lead to industrial accidents, and safety hazards. Designing an appropriate program for maintaining complicated systems is a common concern in academic and professional circles, increasing workplace safety and reducing O\&M costs.

Condition-based maintenance (CBM), which prescribes actions when the actual state of a system is known, has recently received special attention to solving multi-unit maintenance optimization problems. Several CBM models have been designed proposing only the replacement of units, although repair actions are performed with different quality levels. When a unit fails, minimal repair can be performed, which returns it to the same state just before failure (as-bad). The other type of repair, which is referred to as general repair, improves a unit's health, but it does not return it to the as-new condition (Kijima, Morimura and Suzuki, 1988; Pham and Wang, 1996).

Classical sequential decision-making approaches (e.g., dynamic programming (DP)) have been widely used to find the optimal maintenance policy. The advancement of monitoring technologies has motivated researchers to model systems under CBM. However, adding new elements (e.g., condition monitoring variables) to the state space increases the problem's dimensionality, and the DP method may not be practical to solve large-scale problems. For instance, Jafari et al. (Jafari, Naderkhani and Makis, 2018) proposed a threshold-based CBM for a multi-unit system, in which perfect repair is allowed only. This study employed DP to find the optimal policy for maintaining a system where only a core unit is under condition monitoring. The other significant unit is subject to an age-based policy; however, the condition of this unit could have 
been monitored. The other challenge of maintenance optimization of multi-unit assets is the inclusion of repair actions which may cause the curse of modeling when classical methods are applied. Some recent studies proposed policies to evaluate the performance of repair-based policies (Zheng, Zhou and Zhang, 2020). Najafi, Zheng and Lee (2021) studied a series system where condition monitoring and age information are available for units 1 and 2, respectively, and repair actions can be performed on unit 1 . The current paper extends this study, considering the condition monitoring data of both units and applying a methodology with a higher practical value than the DP approach.

AI-based approaches have been recently used to deal with high-dimensional maintenance optimization problems. Yousefi (Yousefi, Tsianikas and Coit, 2020) proposed a CBM policy for a multi-component system subject to deterioration and random shocks. The CBM proposed the repair and replacement actions based on three degradation thresholds, where repair reduces the degradation by one level. The problem is formulated as a Markov decision process, and the Q-learning algorithm is applied to find the optimal policy. Zhang and Si (Zhang and Si, 2020) developed a deep reinforcement learning to learn a policy to minimize the cost of a multi-unit system under CBM when both stochastic and economic dependency exists between units. This study does not allow repair actions, and units can be replaced only.

As discussed above, to the best of our knowledge, no prior research attempted to formulate the maintenance optimization problem of a series system where all units are under condition monitoring in the SMDP framework where repair is allowed. We propose an opportunistic CBM algorithm for a two-unit series system subject to soft and hard failures. The problem is formulated in the SMDP framework, and a reinforcement algorithm is applied to find the optimal policy and the expected long-run cost per unit time. Unlike traditional CBM approaches, no thresholds for maintenance intervention are considered; instead, the proposed policy maps condition monitoring data directly with the repair/replacement actions. Using reinforcement learning enables us to consider both the age and deterioration of the units subject to condition monitoring. Moreover, in this study, general repair is allowed and can be performed at different quality.

\section{System Description}

The system consists of two major units under condition monitoring, and the failure of a unit leads to whole system failure, which is self-announcing. The state space of the system can be expressed as:

$$
\mathrm{S}=\left\{n_{i}, z_{i}, f_{i} \mid n_{i} \in\left\{0, \ldots, \bar{N}_{l}\right\}, \mathrm{z}_{\mathrm{i}} \in\left\{0, \ldots, \bar{Z}_{l}\right\}, f \in\{0,1\}, i \in\{0,1\}\right\}
$$

where $n_{i}, z_{i}$ and $f_{i}$ represent the age, deterioration, and status of unit $i \in\{0,1\}$, respectively. $N_{i}$ indicates the maximum useful age of unit $i, \bar{Z}_{l}$ is the maximum deterioration of unit $i$, and $f$ represents the operational (0) or failure (1) status of the system. It should be noted that the age and deterioration of each unit is discretized into integer values to be able to formulate the problem in discrete state space SMDP framework. The system is inspected at the time interval of $\Delta$, where the condition of each unit is known. Actions $\left(a_{1}, a_{2}\right)$ are performed on unit 1 and unit 2, respectively, where $a_{\mathrm{i}} \in\left\{0, \ldots, Z_{i}\right\} \cup\{\mathrm{MR}\}$ and actions consist of doing nothing $(0)$, general repair $\left(a_{\mathrm{i}} \in\left\{1, \ldots, Z_{i}-1\right\}\right)$, minimal repair (MR), and replacement $\left(Z_{i}\right)$.

The hazard rate is widely applied for condition monitoring purposes. The proportional hazards model (PHM) estimates the risk of failure, integrating the effect of both age $(\mathrm{t})$ and deterioration $\left(X_{t}\right)$. The general form of the PHM can be represented as follows (Cox, 1972):

$$
h\left(t, X_{t}\right)=h_{0}(t) \psi\left(X_{t}\right)
$$

where $h_{0}(t)$ is the baseline function and depends on age only, and $\psi\left(X_{t}\right)$ is a function depending on environmental factors or health data referred to as covariates.

A Weibull PHM distribution is applied to estimate the hazard rate:

$$
h\left(t, X_{t}\right)=\frac{k t^{k-1}}{\lambda^{k}} \cdot \exp \left(\theta X_{t}\right)
$$


where $\lambda$ is the scale parameter, $k$ is the shape parameter, and $\theta$ is the regression coefficient vector of the covariates.

\subsection{Sojourn Times}

Different from soft failure that yields defective products, hard failure occurs randomly and stops the unit from operation. The remaining useful life of units depends on both age and deterioration and is estimated by:

$$
\widehat{L}(t)=\hat{E}(T-t \mid T \geq t)
$$

The remaining useful life can be estimated using the PH function (Liao, Zhao and Guo, 2006):

$$
\hat{L}(t) \approx \int_{0}^{\infty} \exp \left(-\int_{t}^{t+\tau} h\left(s \mid z_{t}\right) d s\right) d \tau
$$

When no action is performed on each unit, the sojourn time is computed as $v_{\mathrm{t}}=\min \left\{\widehat{L}_{l}(t)\right\}$, where $\widehat{L}_{l}$ is the remaining useful life of unit $i \in\{1,2\}$. If $v_{\mathrm{t}}<\Delta$, it means that there is at least one unit whose remaining life, which is computed at the beginning of an inspection interval, is less than $\Delta$. In other words, at least one unit will not survive, and a hard failure occurs over the inspection interval. If $v_{t} \geq \Delta$, both units will survive over the inspection epoch, as their remaining useful lives exceed $\Delta$. For example, let $\widehat{L_{1}}(t)=2, \widehat{L_{2}}(t)=$ 10 , and $\Delta=8$ days, then unit 1 will fail before the inspection interval, and the subsequent decision epoch coincides with unit 1 failure. However, if $\widehat{L_{1}}(t)=12, \widehat{L_{2}}(t)=10$, and $\Delta=8$, both units are operational after $\Delta$ time, and the subsequent decision epoch happens on the inspection interval.

When a maintenance action is performed, sojourn time is computed using the following time parameters:

\begin{tabular}{cc}
\hline \hline Notation & Definition \\
\hline$T_{P G i}(\delta)$ & preventive/opportunistic general repair time of unit $i$ \\
$T_{P R i}$ & preventive/opportunistic replacement time of unit $i$ \\
$T_{H R i}$ & corrective replacement time of unit $i$ when hard failure occurs \\
$T_{S R i}$ & corrective replacement time of unit $i$ when soft failure occurs \\
$T_{H G i}(\delta)$ & corrective general repair time of unit $i$ when hard failure occurs \\
$T_{S G i}(\delta)$ & corrective general repair time of unit $i$ when soft failure occurs \\
$T_{M i}$ & minimal repair time of unit $i$ \\
\hline
\end{tabular}

Table 1. time parameters notation and definition

where $\delta$ is the improvement made by general repair that reduces the unit deterioration from $z$ to $z^{\prime}$. Preventive general repair time is a function of the repair level, and the higher the improvement level, the longer the maintenance duration:

$$
T_{P G i}(\delta)=\left(\frac{\delta}{Z_{i}} \cdot j\right) \cdot T_{P R i}
$$

To what extent repair time is proportional to the replacement time depends on the system structure and is captured by the repair time coefficient $0<j \leq 1$. The other repair times are computed similarly, but corrective general repair times when soft and hard failure occur are proportional to $T_{S R i}$ and $T_{H R i}$, respectively.

\subsection{Reward structure}

In this study, O\&M costs are modeled as negative rewards, and the following cost parameters are considered: 


\begin{tabular}{cc}
\hline \hline Notation & Definition \\
\hline$C_{P G i}(\delta)$ & preventive/opportunistic general repair cost of unit $i$ \\
$C_{P R i}$ & preventive/opportunistic replacement cost of unit $i$ \\
$C_{H R i}$ & corrective replacement cost of unit $i$ when hard failure occurs \\
$C_{S R i}$ & corrective replacement cost of unit $i$ when soft failure occurs \\
$C_{H G i}(\delta)$ & corrective general repair cost of unit $i$ when hard failure occurs \\
$C_{S G i}(\delta)$ & corrective general repair cost of unit $i$ when soft failure occurs \\
$C_{M i}$ & minimal repair cost of unit $i$ \\
$C_{S}$ & system setup cost \\
$C_{L}$ & production loss per unit \\
$C_{I}$ & inspection cost \\
\hline
\end{tabular}

Table 2. cost parameters notation and definition

When no action is taken, no cost is incurred; otherwise, the system costs depend on the action type. For example, the preventive general repair cost is calculated using the following equation:

$$
C_{P G i}(\delta)=C_{S}+C_{I}+\left(\frac{\delta}{Z_{i}} \cdot h\right) \cdot C_{P R i}+C_{L} \cdot T_{P G i}(\delta)
$$

Repair costs are a function of improvement level, and higher quality actions incur higher maintenance costs. However, repair costs are also influenced by other technical factors and repair complexity, which is incorporated by the repair cost coefficient $0<h \leq 1$.

\section{Proposed Policy}

The system is inspected on equidistant intervals of $\Delta$, where the condition of each unit is monitored to obtain the deterioration of each unit. If deterioration of unit $i$ exceeds its soft failure threshold $m_{i}$, a maintenance action is performed to return the deterioration to somewhere below the threshold. Otherwise, a preventive action can be performed to replace or improve the system condition. When hard failure occurs, the failed unit is rectified immediately, and there is an opportunity to improve the other unit.

\section{Reinforcement learning algorithm}

This study considered the long-run average cost optimality criterion and used the SMART (Semi-Markov Average Reward Technique) algorithm to find the optimal policy (Mahadevan et al., 1997). The SMART algorithm learns the value of action $a$ in particular state $s$ under policy $\pi$, i.e., $R(s, a)$, which is derived from the Bellman optimality equation for average reward SMDPs:

$$
R_{\pi}(s, a)=r(s, a)-\rho_{\pi} \cdot v(s, a)+\sum p_{s, s^{\prime}}(a) \max _{a^{\prime}}\left\{R_{\pi}\left(s^{\prime}, a^{\prime}\right)\right\}
$$

where $p_{s, s^{\prime}}(a)$ is the transition probability from state $s$ to $s^{\prime}$ when action $a$ is chosen, and $\rho_{\pi}$ is the average reward. The algorithm updates the utility of action values using temporal differences between the current and next state actionvalues. The above equation can be rewritten as follows if the expected reward $r$ and the expected sojourn time $v$ is replaced with their observed values:

$$
R_{\pi}(s, a) \stackrel{\alpha_{i}}{\leftarrow} r_{i m m}\left(s, a, s^{\prime}\right)-\rho_{\pi} \cdot v_{i m m}\left(s, a, s^{\prime}\right)+\max _{a^{\prime}}\left\{R_{\pi}\left(s^{\prime}, a^{\prime}\right)\right\}
$$

Where $r_{i m m}\left(s, a, s^{\prime}\right)$ and $v_{i m m}\left(s, a, s^{\prime}\right)$ are the observed immediate reward and sojourn time resulted from taking action $a$ in state $s$, and $\alpha_{i}$ is the learning rate at time $i$. The average reward is estimated as the ratio of cumulative immediate reward to the cumulative sojourn times:

$$
\rho=\frac{\sum_{i=0}^{n} r_{\text {imm }}\left(s, a, s^{\prime}\right)}{\sum_{i=0}^{n} v_{\text {imm }}\left(s, a, s^{\prime}\right)}=\frac{R_{\text {Total }}}{T_{\text {Total }}}
$$


The SMART algorithm is as follows, where $\alpha_{i}$ and $\epsilon_{i}$ are the learning and the exploration rates that decay according to a Darken-Chang-Moody search-then-converge procedure (Darken, Chang and Moody, 1992).

1. Set maximum time step $I_{\text {Max }}$ and initialize action values $R_{\text {new }}(s, a)=0$. Set the initial state $(\mathbf{0 , 0 , 0})$, total reward $R_{\text {Total }}=0$, total time $T_{\text {Total }}=0$, and average reward $\rho=0$.

2. While $i<I_{\text {Max }}$ do:

2.1. Choose a random action with probability $\epsilon_{i}$; otherwise, execute action $a$ that maximizes $R_{i}(s, a)$.

2.2. Execute action $a$ and let the next state $s^{\prime}$, generate sojourn time $v_{i m m}\left(s, a, s^{\prime}\right)$ and immediate reward $r_{\text {imm }}\left(s, a, s^{\prime}\right)$.

2.3. Obtain $R_{\text {new }}(s, a)$ following the update rule:

$$
R_{\text {new }}(s, a) \stackrel{\alpha_{i}}{\leftarrow}\left(1-\alpha_{i}\right) R_{\text {old }}(s, a)+\alpha_{i}\left\{r_{\text {imm }}\left(s, a, s^{\prime}\right)-\rho . v_{\text {imm }}\left(s, a, s^{\prime}\right)+\max _{a^{\prime}}\left(R_{\text {old }}\left(s^{\prime}, a\right)\right)\right\}
$$

3. If a nonrandom action is selected in step 2.1:

Update total reward $R_{\text {Total }}$ with $R_{\text {Total }}+r_{\text {imm }}\left(s, a, s^{\prime}\right)$

Update total time $T_{\text {Total }}$ with $T_{\text {Total }}+v_{\text {imm }}\left(s, a, s^{\prime}\right)$

Update average reward $\rho$ with $R_{\text {Total }} / T_{\text {Total }}$.

4. set current state $s$ to the new state $s^{\prime}$ and update $i$ with $i+1$.

\section{Numerical example}

In this section, system parameters, a numerical example, and alternative scenarios are presented. The system consists of two units in series that are both subject to condition monitoring. The lifetime of the units follows Weibull distributions with $\boldsymbol{K}=[30,25] \boldsymbol{B}=[4.2,3.5]$, and $\boldsymbol{\theta}=[1,1.5]$, which represent the scale (months), shape and regression coefficient vectors, where the $i$ th element describes unit $i$. Maximum age, deterioration levels, and soft failure thresholds for the units are 20 months, 8 , and 5, respectively. Setup cost, inspection cost, and production loss are $\$ 50, \$ 20, \$ 10$, respectively, and maintenance costs are represented as follows:

\begin{tabular}{ccc}
\hline \hline Notation & Unit 1 & Unit 2 \\
\hline$C_{P R i}$ & $\$ 4600$ & $\$ 2500$ \\
$C_{H R i}$ & $\$ 5000$ & $\$ 3000$ \\
$C_{S R i}$ & $\$ 4800$ & $\$ 2700$ \\
$C_{M i}$ & $\$ 400$ & $\$ 250$ \\
\hline
\end{tabular}

Table 3. cost parameters

The time parameters are as follows:

\begin{tabular}{ccc}
\hline \hline Notation & $\begin{array}{c}\text { Unit 1 } \\
\text { (month) }\end{array}$ & $\begin{array}{c}\text { Unit 2 } \\
\text { (month) }\end{array}$ \\
\hline$T_{P R i}$ & 0.023 & 0.019 \\
$T_{H R i}$ & 0.033 & 0.029 \\
$T_{S R i}$ & 0.026 & 0.021 \\
$T_{M i}$ & 0.018 & 0.011 \\
\hline
\end{tabular}

Table 4. time parameters

Note that general repair times and costs are computed according to Sections 2.1 and 2.2, respectively. Given the abovementioned parameters, the proposed policy is compared with the replacement policy, in which no repair actions are allowed, and units can be replaced only. In this experiment, the repair time coefficient is $j=1$, and three values for the repair cost coefficient $(h=0.5,0.67$ and 1$)$ are considered. The following graph shows the average reward under the replacement policy and the proposed policy when $h$ takes $1,0.5$, and .67. The inspection interval is three months for all policies. 


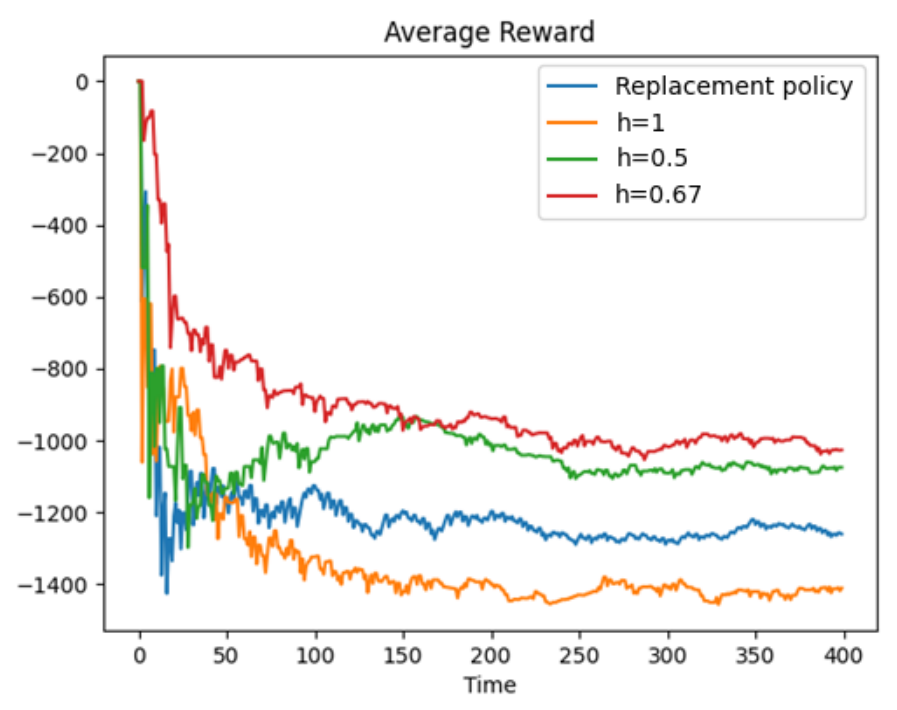

Graph 1. The long-run average cost of repair and replacement

As shown in the graph the repair policy does not necessarily results in a lower long-term average reward than the replacement policy. The replacement policy incurs the average cost of $\$ 1202$ and outperforms the repair policy when $h=1$ with the average cost of $\$ 1398$. However, the repair policy converges to a lower average cost when $h$ decreases to 0.67 and 0.5 , resulting in the average cost of $\$ 925$ and $\$ 995$, respectively. The findings show that the repair policies with $h=0.5$ and $h=0.65$ accomplish $20 \%$ and $23 \%$ cost reductions, respectively, compared to the replacement policy.

\section{Conclusion}

In this paper, an opportunistic maintenance policy with general repair is proposed for a two-unit series system, in which the condition of both units is monitored. The maintenance problem is formulated in the SMDP framework, and the condition under which the proposed maintenance policy minimizes the longrun average cost per unit time is discussed. The policy prescribes preventive, corrective, and opportunistic actions consisting of minimal repair, general repair, and replacement. The novelty of this research is the inclusion of general repair in the maintenance modeling of two-unit systems in the SMDP framework, where repair actions with different quality are performed without considering maintenance thresholds. The proposed model-free reinforcement learning algorithm allowed us to evaluate repair-based policies, while classical approaches may suffer the curse of dimensionality and modeling when applied to such a problem (Encapera, Gosavi and Murray, 2019), and is capable of solving large-scale problems (Mahadevan et al., 1997). A numerical example confirms the superiority of the proposed policy over a similar policy in which general repair is not considered.

To further the research, we plan to study systems with a higher number of units to enhance the practical value of the research, which requires finding an approach capable of managing a large state space. Furthermore, providing an analytical solution for the problem that shows the optimal policy will confirm the validity of the proposed approach.

\section{References}

Cox, D. R. (1972) 'Regression models and life-tables', Journal of the Royal Statistical Society: Series B (Methodological). Wiley Online Library, 34(2), pp. 187-202.

Darken, C., Chang, J. and Moody, J. (1992) 'Learning rate schedules for faster stochastic gradient search', in Neural networks for signal processing. Citeseer. 
Encapera, A., Gosavi, A. and Murray, S. L. (2019) 'Total productive maintenance of make-to-stock production-inventory systems via artificial-intelligence-based iSMART', International Journal of Systems Science: Operations \& Logistics. Taylor \& Francis, pp. 1-13.

Jafari, L., Naderkhani, F. and Makis, V. (2018) 'Joint optimization of maintenance policy and inspection interval for a multi-unit series system using proportional hazards model', Journal of the Operational Research Society. Taylor \& Francis, 69(1), pp. 36-48.

Kijima, M., Morimura, H. and Suzuki, Y. (1988) 'Periodical replacement problem without assuming minimal repair', European Journal of Operational Research. Elsevier, 37(2), pp. 194-203.

Liao, H., Zhao, W. and Guo, H. (2006) 'Predicting remaining useful life of an individual unit using proportional hazards model and logistic regression model', in RAMS'06. Annual Reliability and Maintainability Symposium, 2006. IEEE, pp. 127-132.

Mahadevan, S. et al. (1997) 'Self-improving factory simulation using continuous-time average-reward reinforcement learning', in MACHINE LEARNING-INTERNATIONAL WORKSHOP THEN

CONFERENCE-. MORGAN KAUFMANN PUBLISHERS, INC., pp. 202-210.

Najafi, S., Zheng, R. and Lee, C.-G. (2021) 'An optimal opportunistic maintenance policy for a two-unit series system with general repair using proportional hazards models', Reliability Engineering \& System Safety. Elsevier, p. 107830.

Pham, H. and Wang, H. (1996) 'Imperfect maintenance', European journal of operational research. Elsevier, 94(3), pp. 425-438.

Yousefi, N., Tsianikas, S. and Coit, D. W. (2020) 'Reinforcement learning for dynamic condition-based maintenance of a system with individually repairable components', Quality Engineering. Taylor \& Francis, 32(3), pp. 388-408.

Zhang, N. and Si, W. (2020) 'Deep reinforcement learning for condition-based maintenance planning of multi-component systems under dependent competing risks’, Reliability Engineering \& System Safety. Elsevier, 203, p. 107094.

Zheng, R., Zhou, Y. and Zhang, Y. (2020) 'Optimal preventive maintenance for wind turbines considering the effects of wind speed', Wind Energy. Wiley Online Library, 23(11), pp. 1987-2003. 